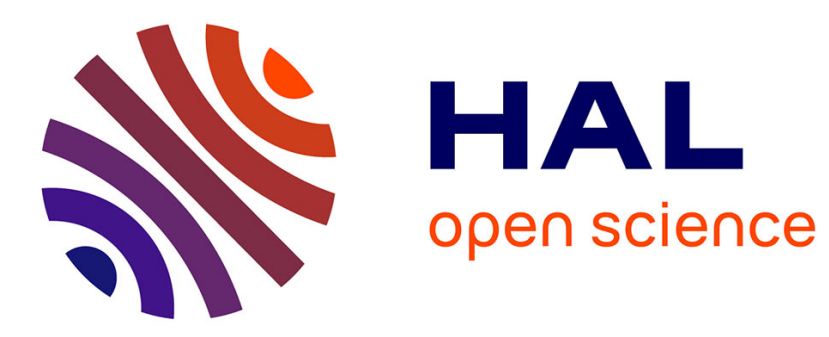

\title{
Aller et venir : des verbes de déplacement aux auxiliaires aspectuels-temporels-modaux
}

\author{
Jacques Bres, Emmanuelle Labeau
}

\section{To cite this version:}

Jacques Bres, Emmanuelle Labeau. Aller et venir: des verbes de déplacement aux auxiliaires aspectuels-temporels-modaux. Langue française, 2013, 179, pp.13-28. hal-00948300

\section{HAL Id: hal-00948300 \\ https://hal.science/hal-00948300}

Submitted on 27 Jun 2018

HAL is a multi-disciplinary open access archive for the deposit and dissemination of scientific research documents, whether they are published or not. The documents may come from teaching and research institutions in France or abroad, or from public or private research centers.
L'archive ouverte pluridisciplinaire HAL, est destinée au dépôt et à la diffusion de documents scientifiques de niveau recherche, publiés ou non, émanant des établissements d'enseignement et de recherche français ou étrangers, des laboratoires publics ou privés. 


\title{
RESUME
}

Aller et venir : des verbes de déplacement aux auxiliaires aspectuels-temporels-modaux Après avoir décrit le fonctionnement des verbes aller et venir, nous développons l'hypothèse selon laquelle leur grammaticalisation en auxiliaires s'effectue sur la base de l'élément spatial qui structure tant leur sémantisme commun - le déplacement vers une destination - que leur différence : l’orientation déictique (venir) / non déictique (aller) du déplacement. Nous testons cette hypothèse en analysant les principaux emplois des périphrases de structure [aller / venir (+ prép.) + V(infinitif, gérondif)].

Mots-clefs : aller, venir, auxiliaire, grammaticalisation, déplacement, déictique

\begin{abstract}
Aller and venir : from motion verbs to aspectual-temporal-modal auxiliaries

After a description of how motion verbs aller and venir work, we develop an hypothesis according to which their grammaticalisation into auxiliaries relies on the spatial element that structures their shared semantics - movement towards a destination - as well as their distinctive feature : a deictic (venir) or non deictic (aller) orientation of the movement. We test that hypothesis against an analysis of the main uses of periphrases in the [aller / venir (+ prep.) + V(infinitive, gerund)] pattern.
\end{abstract}

Keywords : aller, venir, auxiliary, grammaticalization, movement, deixis

JACQUES BRES, Praxiling, UMR 5267 CNRS-Montpellier III, EMMANUELLE LABEAU, Aston University

\section{Aller et venir : des verbes de déplacement aux auxiliaires aspectuels-temporels-modaux ${ }^{1}$}

Que reste-t-il du sens spatial des verbes de déplacement aller et venir dans leurs nombreux emplois en tant qu'auxiliaires dans les tours [aller / venir (+ prép.) + V(infinitif, gérondif, participe passé)] ? Cet article fait l'hypothèse que la grammaticalisation (i. a. Meillet 1912/1926, Hopper et Traugott 1993/2003, Heine 2003, Narrog et Heine 2011) de ce couple de verbes en auxiliaires aspectuels-temporels-modaux s'effectue sur la base de l'élément spatial $^{2}$ qui structure tant leur sémantisme commun - le déplacement vers une destination que leur différence : l’orientation déictique (venir) / non déictique (aller) du déplacement.

Le fait que de nombreuses langues du monde développent des tours périphrastiques à partir des formes itive ('aller') et ventive ('venir') a donné matière à de nombreux travaux de linguistique générale (i. a. Fleischman 1982a, Bourdin 1992, Hagège 1993, Bybee et al. 1994, Dahl 2000) ; de linguistique romane (i. a. Fleischman 1982b, Lamiroy 1983, Squartini 1998) ; de linguistique française (i. a. Gougenheim 1929, Damourette et Pichon 1911-1936/1970, Bres et Labeau 2012a). Différentes recherches ont été consacrées, pour ce qui est du français, aux emplois de aller (i. a. Larreya 2005, Lansari 2009) ou de venir (i. a. Bourdin 1999, 2005, Honeste 2005). La spécificité du présent travail tient à ce qu'il s'attache à montrer contrastivement la rémanence du sens spatial de ces deux verbes dans les différents effets de sens aspectuels-temporels-modaux des tours où ils se voient grammaticalisés en auxiliaires.

\footnotetext{
${ }^{1}$ Nous remercions S. Azzopardi, S. Sarrazin, C. Garabato ainsi que les deux lecteurs anonymes de la revue : leurs remarques nous ont permis d'améliorer une première version de ce texte.

${ }^{2}$ Pour une analyse qui ne place pas le sens de déplacement à la base de ces verbes, cf. Bouchard 1993.
} 
Pour ce faire, nous commencerons par décrire la sémantique spatiale de aller et de venir en tant que verbes de déplacement. Nous établirons dans un second temps comment, au terme du processus de grammaticalisation de ces verbes en auxiliaires, perdure, abstraite, cette sémantique sur laquelle se sont développés différents effets de sens repérables en discours.

L'analyse repose sur un corpus étoffé de 3000 occurrences relevé dans l'oral conversationnel, dans l'écrit littéraire et journalistique et sur internet. Pour des raisons d'espace, nous ne nous intéresserons qu'aux tours périphrastiques [aller / venir (+ prép.) + V(infinitif, gérondif)], ce qui exclut les formes avec en, du type s'en aller / venir + V(infinitif, participe passé).

\section{Description de aller et venir en tant que verbes de déplacement}

Différentes descriptions du couple de verbes de déplacement aller / venir ont été proposées en français (i. a., Damourette et Pichon op. cit., Kerbrat-Orecchioni 1980). Nous nous appuierons plutôt sur l'analyse de Chevalier $1976^{3}$, qui nous paraît allier robustesse et finesse. Nous distinguerons deux éléments : le mouvement de déplacement, et son orientation.

Le déplacement dans l'espace extérieur signifié par ces verbes est analysable comme le parcours par l'actant sujet d'un «espace fait de positions successives » (Chevalier op. cit. : 265) entre un lieu initial (l'origine $\alpha$ ) et un lieu final du déplacement (la destination $\omega$ ). Ces deux lieux n'ont pas le même statut : en tant que verbes «à changement de relation final avec déplacement antérieur intégré » (Aurnague 2008 : 1912), aller et venir impliquent plus fortement la borne de destination $\omega$ que la borne d'origine $\alpha$.

Ces bornes sont, par ailleurs, prises en compte différemment par aller et par venir :

$$
\text { aller : ] } \alpha \rightarrow \omega[\quad \text { venir : ] } \alpha \rightarrow \omega]
$$

Aller représente le déplacement comme un intervalle ouvert à gauche (origine) comme à droite (destination) ; venir représente le déplacement comme un intervalle ouvert à gauche mais fermé à droite. La fermeture de venir à droite tient à la présence d'une subjectivité : la destination $\omega$ est le lieu où se situe /se situait/ se situera l'énonciateur principal et / ou l'énonciataire, ou un point de vue subjectif à partir duquel s'organise l'espace.

Venir est un verbe déictique (Fillmore 1997 (1967/1975)), à savoir que le déplacement est orienté vers une subjectivité dont l'actant se rapproche (afférence) ; aller est, quant à lui, un verbe non déictique.

L'appréhension de aller comme signifiant un déplacement non-déictique nécessite quelque précision. Damourette et Pichon considèrent que « aller exprime une efférence, c'est-à-dire un 'sens de marche' qui s’éloigne du moi-ici-maintenant » (op. cit.: § 1646, p.101). Ils attribuent à aller l'expression d'un mouvement inverse de celui de venir, selon l'opposition éloignement / rapprochement du centre déictique. Cette analyse, partagée par de nombreux auteurs, achoppe sur deux pierres: certaines occurrences ne peuvent être analysées en tant qu'éloignement d'un centre déictique (infra (6-7) ; certains faits analysés infra ne peuvent s'expliquer que parce que aller n'est pas concerné par la catégorie de la deixis.

En parlant de aller comme verbe non déictique, nous entendons que le déplacement qu'il prend en charge n'est pas situé par rapport à une subjectivité (Chevalier 1976 parle de déférence), ce qui peut co(n)textuellement, par inférence, signifier un éloignement du centre déictique.

On dira que, eu égard à la déicticité, venir est la forme marquée ; aller, la forme non marquée. Comme nous l'avons dit, le centre déictique peut être un point de vue : le locuteur délègue alors sa subjectivité à un actant [+ humain], notamment, en textualité narrative, à un

\footnotetext{
${ }^{3}$ Consacrée au fonctionnement contrastif des formes itive et ventive en français et en espagnol.
} 
personnage comme en (1) où le narrateur adopte le point de vue de Léon qui attend impatiemment la venue de son amante dans la cathédrale de Rouen :

(1) Il marchait auprès des murs. Jamais la vie ne lui avait parue aussi bonne. Elle allait venir tout à l'heure, charmante, agitée. (Flaubert G., Madame Bovary, 1856)

Lorsque la destination est un objet, qui ne soit pas un lieu où puisse siéger le locuteur ou l'allocutaire, c'est aller qui est en principe sollicité (2) :

(2) István va à la fenêtre, il jette un coup d'oeil, la main en visière. (Garat A-M., István arrive par le train du soir, 1999)

Mais rien n'interdit que la subjectivité puisse se glisser même sous ce type de destination objectale :

(3) Boucard avait fini son addition. Il sentit l'odeur de son chocolat, quitta son fauteuil de canne, vint à la cheminée, toisa le vieil homme, regarda le carrick et fit une grimace indescriptible (Balzac H., Le Colonel Chabert, 1844)

Le narrateur pose un œil, tel une caméra, sur la cheminée, à partir duquel l'actant est vu se déplacer vers cet objet.

Cette structuration différente de la destination $\omega$ du déplacement rend compte des comportements syntaxiques suivants de chacun des deux verbes, dont nous verrons l'importance pour les emplois en tant qu'auxiliaires :

(i) venir ne demande pas l'explicitation de la destination par un complément, alors qu'aller tend à l'exiger : (4) est tout aussi bon que (4a) ; (5) fait difficulté, difficulté que lève l'explicitation de la destination (5a) :

(4) Corinne vient ce soir.

(4a) Corinne vient au théâtre ce soir.

(5) *Corinne va ce soir.

(5a) Corinne va au théâtre ce soir.

Du fait de son fonctionnement déictique - bornage à droite du déplacement - venir peut en discours ne pas expliciter la destination (4), puisqu'elle correspond implicitement au lieu où se place un centre déictique.

Du fait de son fonctionnement non déictique, aller est ouvert à droite ${ }^{4}$, ce qui rend compte de l'inacceptabilité de (5) dans la mesure où la destination du déplacement n'est pas actualisable - on a envie de demander où va Corinne. Ajoutons toutefois que, en discours, aller peut sporadiquement, en toute acceptabilité, ne pas expliciter la destination : le sens produit est alors celui d'un déplacement sans destination, ce qui peut en contexte signifier la force du destin qui entraîne on ne sait où (6), le voyage sans limite ni destination de l'amour (7), etc. :

(6) Tu me crois peut-être, / Un homme comme sont tous les autres, un être / Intelligent, qui court droit au but qu'il rêva. / Détrompe-toi. Je suis une force qui va ! (Hugo V., Hernani, 1830) ${ }^{5}$

\footnotetext{
${ }^{4}$ Les deux traits qui en français sont concomitants ne le sont p. ex. pas en espagnol : la forme itive ir a la structure ] $\alpha \rightarrow \omega$ ], c'est-à-dire que le parcours est borné à droite, sans pour autant que cette borne soit le lieu d'une subjectivité ; ce qui permet l'emploi de ir sans complément : « ya voy ! (littéralement 'je vais' : ‘je viens tout de suite !').

${ }^{5}$ Hernani proclame, deux vers plus avant : « Où vais-je ? Je ne sais. Mais je me sens poussé / D'un souffle impétueux, d'un destin insensé. »
} 
(7) Emma ne dormait pas. (...) Au galop de quatre chevaux, elle était emportée depuis huit jours vers un pays nouveau, d'où ils ne reviendraient plus. Ils allaient, ils allaient, les bras enlacés, sans se parler. (Flaubert G., Madame Bovary)

Venir dénote toujours un procès télique, plus précisément un accomplissement, ce que teste le fait qu'il est compatible avec en $x$ temps et incompatible avec pendant $x$ temps :

(8) Corinne vient à l’université en /*pendant 10'

Aller dénote le plus souvent un procès télique, plus précisément un accomplissement, et dans ce cas il a les mêmes (in)compatibilités que venir :

(9) Corinne va à l’université en /*pendant 10’

Mais du fait de sa structure d'intervalle ouvert à droite, il peut, dans des occurrences comme (6-7), dénoter un procès atélique, plus précisément une activité, ce que teste alors sa compatibilité avec pendant $x$ temps :

(7a) Ils allaient, ils allaient, pendant des heures, les bras enlacés, sans se parler.

(ii) Aller, ne marquant pas la destination $\omega$ vers laquelle le déplacement est orienté, ne peut être accompagné du seul complément d'origine $\alpha(10)$, pas plus qu'il n'accepte l'interrogation sur cette origine (10a) ; venir, marquant la destination $\omega$, peut être accompagné du seul complément d'origine $\alpha$ (11), et accepte l'interrogation sur cette origine (11a) :

(10) *Corinne va de Paris.

$(10 \mathrm{a}) * \mathrm{D}$ ’où va Corinne?

(11) Corinne vient de Paris.

(11a) D’où vient Corinne ?

Aller et venir sont deux verbes de déplacement orientés vers la destination $\omega$, qui se différencient par la façon dont ils prennent en compte ce terme. Il apparaît qu'aller, du fait de sa structure ouverte à droite (] $\alpha \rightarrow \omega$ [), qui procède de sa non-déicticité, a plus de contraintes syntaxiques que venir, du fait de sa structure fermée déictiquement à droite (]$\alpha \rightarrow \omega])$. Nous allons voir que cette inégalité s’inverse dans les tours périphrastiques où aller et venir sont auxiliaires : c'est venir qui se voit en partie contraint par sa déicticité, et qui se grammaticalise en moins d'emplois que aller.

\section{Lorsque le verbe se grammaticalise en auxiliaire}

\subsection{Hypothèse}

Nous n'analyserons pas ici selon quel processus aller et venir se grammaticalisent en auxiliaires. Si l'hypothèse de Hopper \& Traugott (1993/2003 :108) est exacte, le chemin de grammaticalisation des lexèmes verbaux est le suivant : [full verb $>$ auxiliary $>$ clitic $>$ affix]. Dans le cas présent, il s’agit de la première étape (auxiliary) : les verbes de déplacement fonctionnent comme des auxiliaires, ce qui se note notamment dans :

- leur structure syntaxique : aller et venir sont suivis non d'un SP complément de lieu (aller / venir à Paris), mais d'un verbe à l'infinitif ou au gérondif. Soit donc : [aller / venir (+ prép.) $+\mathrm{V}$ (infinitif, gérondif)]. Ce critère est cependant loin de suffire : aller et venir suivis d'un verbe à l'infinitif peuvent fonctionner comme verbes de déplacement : 
(12) je suis venu te dire que je m’en vais (Gainsbourg S., titre de chanson, 1975)

(13) Si Bonne-Maman était restée pour passer la nuit, dans la chambre d'amis, il serait allé lui dire « Non, vat'en ». (Navarre Y., Biographie, 1981)

Aussi faut-il ajouter la possibilité suivante, nommée par Hagège «proof by anachrony principle » (1993 : 200-202) : la forme est grammaticalisée s’il y a la possibilité de la faire suivre de la forme lexicale elle-même (introduite ou non par une préposition). Soit les tours [auxiliaire aller + V.aller] et [auxiliaire venir + de + V.venir] :

(14) Nous allons aller par ici. Je connais bien le chemin. (Michaux H., Un certain Plume, 1936)

(15) - ah ! Mon cher, tu arrives mal ; ma maîtresse vient de venir, et il y a quinze jours que je ne l'ai vue ! (Murger H., Scènes de la vie de bohème, 1848)

- leur sémantisme : aller et venir ne signifient plus un déplacement dans l'espace extérieur, mais, en effacement semble-t-il de leur sens spatial, servent à construire des valeurs aspectuo-temporelles et modales, p. ex. l'ultériorité (14) et l'antériorité (15) proximales.

Pour autant, y a-t-il eu effacement, désémantisation du sens spatial ? Non, mais resémantisation aspectuo-temporelle et modale dans laquelle ce n'est plus l'actant sujet (grammatical) qui se déplace selon un mouvement concret, mais le sujet cognitif selon un « mouvement abstrait» 6 .

Ce déplacement abstrait du sujet cognitif se produit vers le procès qui suit l'auxiliaire, ou à partir de lui :

- vers le procès, qui correspond à la destination $\omega$, lorsque la construction est directe ou indirecte (prépositions à et jusqu'à) ; et dont le temps interne peut être saisi globalement à partir de sa borne initiale par l'infinitif, ou en un point de son cours par le gérondif. Soit donc les tours : [aller / venir + (à, jusqu'à) + Vinf.] ; [ aller + Vgérondif] ;

- à partir $d u$ procès, qui correspond à l'origine $\alpha$, lorsque ledit procès est introduit par la préposition de, dans le tour [venir de + Vinf.].

Ce mouvement abstrait conserve la déicticité de venir, la non-déicticité d'aller.

En résumé : dans les emplois en auxiliaires du couple aller-venir persiste le sens de déplacement déictiquement orienté (venir) ou non (aller), mais abstrait, pour produire les effets de sens aspectuo-temporels et modaux. C'est même à partir de cette base que peuvent s'expliquer tant les différents tours dans lesquels aller et venir fonctionnent comme auxiliaires que les différences dans leurs possibilités d'emploi. C'est ce que nous allons développer maintenant.

\subsection{Les différents effets de sens}

Dans nos recherches antérieures (Bres et Labeau 2012a), nous avons relevé 9 effets de sens produits par la structure [aller / venir (+ prép.) + V(infinitif, gérondif)] : 4 sont spécifiques de aller (le narratif, l'ultérieur, le modalisateur, le progressif); 2 de venir (l'antérieur immédiat, l'accidentel) ; 3 sont communs aux deux auxiliaires (l'extraordinaire, l'extrême, l'illustratif) ${ }^{7}$.

\footnotetext{
${ }^{6}$ Langacker $1987: 76$ : «Ce n’est pas le sujet qui bouge dans le temps quand un phrase comme (28) [il va ouvrir la porte] a le sens du futur, mais plutôt celui qui conceptualise la situation, dont le mouvement est à la fois abstrait et subjectif ».

${ }^{7}$ Compte tenu de l'espace dont nous disposons, nous faisons le choix de ne traiter ni l'accidentel ni l'illustratif (cf. Bres et Labeau 2013a).
} 


\subsubsection{Effets de sens spécifiques}

\section{Le narratif et l'ultérieur}

(16) Dès le soir mesmes, elle envoya prier le gentil homme de la venir veoir la nuict. Mais le mary, qui estaoit si tourmenté de jalousie qu'il ne pouvoit dormir, vat prendre une cape et s'en vat frapper à la porte du logis de sa femme. (Marguerite de Navarre, Heptaméron)

(17) Par expérience perso, elle va le quitter mais restera pas longtemps avec toi. (www.forum-auto.com)

Comment rendre compte de ce que aller au présent + Vinf. puisse concurrencer en emploi narratif (16), un passé simple, temps de l'époque passée (vat prendre/prit); et en emploi d’ultériorité (17), le futur synthétique, temps de l’époque future (va le quitter / le quittera) ? Cette apparente incohérence se résout si l'on considère que, dans les deux cas, aller signifie un mouvement abstrait vers la borne initiale du verbe à l'infinitif.

En (16), nous sommes en énonciation historique et dans le cadre de la séquentialité narrative, c'est-à-dire une textualité de type iconique : l'ordre des propositions narratives (du premier plan) tend à (re)produire l'ordre des événements selon lequel le temps progresse $(\mathrm{a}<\mathrm{b}<\mathrm{c})$ : en appui sur et à partir du précédent procès au passé simple (envoya), va pose un mouvement qui conduit prospectivement au procès suivant (prendre). La périphrase va prendre est purement aspectuelle ${ }^{8}$. C'est seulement le contexte - en (16) notamment le passé simple envoya et les imparfaits était tourmenté et pouvait - qui permet de comprendre que le procès prendre actualisé par va est de fait situé dans l'époque passée.

Ce tour se développe entre la fin du XIII ${ }^{\text {ème }}$ et le XVI ${ }^{\text {ème }}$ siècle, et disparaît au XVII ${ }^{\text {ème }}$. Alternant avec des prétérits synthétiques, il fonctionne comme une ressource stylistique complémentaire du présent narratif pour dynamiser le récit. On rencontre également ce même type d'emploi dans les langues de la Romania de l'Ouest de caractère gallo-roman - ancien espagnol, catalan, occitan. C'est seulement en catalan et dans certains dialectes occitans (gascon) que le tour devient un temps de l'époque passée, à savoir un prétérit périphrastique, qui tend à éliminer le prétérit synthétique (Colón 1976, Bres et Barceló 2007).

En (17), nous sommes en énonciation de discours (forum sur internet). La périphrase alterne avec la forme synthétique du futur: "elle va le quitter mais restera pas longtemps ». Le mouvement de prospection vers la borne initiale du procès à l'infinitif qui suit s'opère à partir du $t_{0}$ de l'énonciation, du fait du fonctionnement contextuellement déictique du présent. Ce qui conduit à localiser le procès dans une époque ultérieure à $t_{0}$, c'est-à-dire l'époque future ; et plus précisément l'époque future proche, ce que nous expliquons infra 4.

Diachroniquement, l'emploi d'ultériorité est plus tardif que l'emploi narratif : sporadique au $\mathrm{XIII}^{\text {ème }}$, il se répand au $\mathrm{XV}^{\text {ème }}$ dans des textes plus vulgaires que ceux où apparaît l'emploi narratif, notamment dans les dialogues. Aux $X^{\text {eme }}-X^{\text {ème }}$, on trouve parfois des occurrences dans lesquelles cohabitent les deux emplois, narratif et ultérieur :

(18) Le maistre de ceans [...] lui va dire: «Viens ça, Morthemer, je te vas faire un marché » (Nicolas de Troyes, Le Grand parangon des nouvelles nouvelles, 1535, cité par Gougenheim op. cit.: 99).

avec la répartition systématique suivante : emploi narratif pour le récit en $3^{\text {ème }}$ personne («Le maistre de ceans luy va dire »); emploi d'ultériorité pour le dialogue rapporté (« je te vas faire un marché »). Progressivement néanmoins, l’emploi d’ultériorité se développe, se normalise,

\footnotetext{
${ }^{8}$ Ce qui explique que certains (Gougenheim 1929, Berchem 1969) aient pu parler, par simplification, d'aspect inchoatif.
} 
et élimine l'emploi narratif, qui n’apparaît plus guère, dans le premier tiers du XVII ${ }^{\text {ème }}$, que dans des textes de caractère populaire, avant de devenir un archaïsme ${ }^{9}$.

Le paradoxe pointé initialement - va comme auxiliaire des époques passée et future - se trouve résolu: $v a$, en grammaticalisation de son sens spatial, signifie un mouvement de prospection vers la destination, à savoir le procès à l'infinitif, mouvement qui selon le cotexte se densifie en effet de sens de narration (16), ou d'ultériorité (17).

Nous avons expliqué en quoi aller, de par le mouvement de prospection qui lui est commun à venir, rendait compte des emplois narratif et d'ultériorité. Qu'en-est-il de sa non-déicticité ? Venir n'était-il pas à même de participer à la production de ces tours ? On distinguera les deux emplois :

- Pour ce qui est de l'emploi narratif, on peut avancer que la progression narrative consiste à se déplacer d'un procès à l'autre, sans faire le détour par une instance énonciative, ce que permet la non-déicticité de aller. Les autres langues romanes confirment cette hypothèse : l'ancien espagnol, le catalan, l'occitan produisent l'effet de sens narratif à partir de la forme itive ; aucune ne le fait à partir de la forme ventive. Il convient toutefois d'être prudent dans nos conclusions : Bourdin 2008 établit à partir d'un échantillon important de langues du monde, notamment africaines, que la forme ventive est tout aussi sollicitée que la forme itive pour construire ce type d'emploi (qu'il nomme sequentiality). Ajouter venir à : vinrent à passer

- Plus de prudence encore s’impose pour la mise en relation du paramètre de la (non)déicticité avec l'emploi d'ultériorité. Si l'occitan, le portugais, l'espagnol (avec le concours de la préposition $a$ ), l'anglais (avec le concours de la préposition to) ont développé un emploi d'ultériorité à partir de l'itif, le romanche parmi les langues romanes, ainsi que les langues scandinaves (danois, norvégien, suédois et marginalement finnois, Dahl 2000) l'ont développé à partir du ventif. Bybee et al., dans leur échantillon de 94 langues appartenant à différents groupes linguistiques, en relèvent 10 dont le futur remonte à aller et autant dont le futur a évolué à partir de venir (1994: 252-3). Typologiquement, venir n’est pas allergique à l'ultériorité. On dira simplement que le français, usant de aller, conçoit l'ultérieur comme une destination où l'on va et non où l'on vient, à savoir qu'il ne place pas en $\omega$ un centre déictique.

\section{Le modalisateur}

On sera beaucoup plus bref pour l'analyse de l'emploi modalisateur dans lequel aller est suivi du verbe dire: on va dire, je vais dire, j'allais dire, etc. (Lansari 2010, Labeau 2012a, Steuckardt 2012) qui s’est développé au XX ${ }^{\text {ème }}$ :

(20) elle est un peu à l'ouest on va dire (conversation)

Cet emploi est dérivé de l'emploi d’ultériorité : il consiste à modaliser autonymiquement un segment de l'énoncé (en (20), “à l'ouest ») en plaçant son énonciation en ultériorité, ce qui revient à bémoliser sa prise en charge ${ }^{10}$. L'ultériorité se signifiant en français par aller, la modalisation qui en dérive usera également de la forme itive.

\footnotetext{
${ }^{9}$ Le français développera au XX ${ }^{\text {ème }}$, à partir de l'emploi d'ultériorité, un nouveau tour narratif apparemment de même forme, mais fonctionnant quelque peu différemment puisqu'aller peut s'y conjuguer, en plus du présent, à l'imparfait (Bres et Labeau 2012c, Labeau 2012b) :

(19) Teddy Pendergrass est remarqué par Harold Melvin, leader du quintette vocal The Blue Notes. Il rejoint alors la formation, qui va enchaîner une succession de tubes. (Le Monde, Obituaire de T. Pendergrass, $27.1 .2010)$

${ }^{10}$ Les grammairiens parlent, pour cet effet de sens également produit au futur synthétique (on dira, etc.), de futur de mitigation.
} 


\section{Le progressif}

(21) L'automne va mourant, / en souriant. (Guérin M., Poèmes, 1839)

Il s'agit du seul tour où l'auxiliaire est suivi non de l'infinitif, mais du gérondif. A la différence de l'infinitif qui saisit le temps interne du procès globalement de sa borne initiale à sa borne finale, le gérondif le donne à voir dans son cours, après sa borne initiale et avant sa borne terminale, de façon sécante. L'effet de sens aspectuel produit est celui du progressif : ce qui est représenté, c’est le procès qui, saisi dans son cours (gérondif), d'instant en instant progresse dans sa réalisation $(v a)$. Le mécanisme de production nous semble être le suivant: le mouvement dont est porteur aller a pour destination non la borne initiale du procès (infinitif), mais un point de son cours (gérondif). Aller ne peut donc signifier le mouvement abstrait vers ce procès, à savoir la prospection, puisque ce procès, déjà atteint, est saisi cursivement: le mouvement s'applique à l'en-cours lui-même, qu'il dynamise. Le procès mourir en (21) est représenté comme se réalisant d'instant en instant: à savoir dans sa progression.

On sait que ce tour fréquent en ancien et moyen français, s’étiole à partir du XVII ${ }^{\text {ème }}$, pour ne plus subsister que comme archaïsme. Venir pouvait-il participer à sa production ? Schøsler (2007) semble le penser qui propose, parmi différentes occurrences avec aller, une (seule) occurrence de venir :

(22) si commanda a sonner la retraicte, Jehan et Girard venoyent chevaychant derriere affin que de leurs gens ne perdeissent (...) (Gilion de Trasignyes, $\left.\mathrm{XV}^{\mathrm{ème}}\right)$

Elle ajoute qu’on ne saurait « discerner une différence de sens » (op. cit. : 96) entre aller et venir.

L'exemple proposé nous semble peu probant : venir peut aussi ici être analysé comme verbe de déplacement, le gérondif « chevauchant derrière » explicitant la façon dont se déroule ledit déplacement ('ils venaient en chevauchant') ${ }^{11}$.

Il nous semble donc que - sous bénéfice d'inventaire - c'est aller qui en français a servi à construire le progressif, sans que l'on puisse tirer de conclusion solide sur ce choix : l'espagnol p. ex., qui fait un usage fréquent du progressif, l'a développé sur aller comme sur venir, en différenciant d'ailleurs leurs conditions d’emploi (García Fernández 2006).

\section{L'antérieur proximal}

L'auxiliaire venir sert, avec l'aide de la préposition de, à signifier l'antériorité proximale d'un procès par rapport à un autre procès (23), ou par rapport au t $t_{0}$ de l'énonciation (24) :

(23) Quand elle reparut, le bateau venait de partir. (Maupassant G., Au printemps)

(24) Coucou, Je viens de me faire plaquer il y a 5 minutes, pour une broutille ! (forum.aufeminin.com/)

Venir de pose le procès partir comme antérieur proximal à reparaître, et le procès se faire plaquer comme antérieur proche de $\mathrm{t}_{0}$. Cet effet de sens nous semble également issu de la grammaticalisation du déplacement avec destination déictique du verbe venir.

\footnotetext{
${ }^{11}$ Gougenheim (op. cit. : 16) précise que dans le tour venir + gérondif, « il y a toujours mouvement réel ».
} 
Sans entrer dans le détail de l'explication de la grammaticalisation particulièrement complexe de ce tour (Bourdin 2005, De Mulder 2010, Vetters 2010, Bres et Labeau 2013b en prép.), nous dirons que :

- comme vu supra en (11), venir verbe, du fait qu'il incorpore la destination $\omega$ de par sa déicticité, peut expliciter, à l'aide de la préposition de, seulement l'origine $\alpha$ du déplacement (Corinne vient de Paris). C'est cette possibilité qu'exploite la périphrase venir de. Aller, du fait de sa non-déicticité qui l'empêche de signifier seulement l'origine du déplacement, ne saurait participer à la production de ce sens (*le bateau allait de partir). Et de fait aucune langue romane qui développe l'antériorité proximale de façon périphrastique ne le fait sur aller : l'occitan use de venir comme le français ; les langues ibéro-romanes catalan, espagnol, portugais - ont grammaticalisé en auxiliaire un autre verbe : acabar ('achever', 'finir') ;

- du fait de la préposition de, le déplacement abstrait du sujet cognitif se fait non plus vers la destination $\omega$, mais à partir de l'origine $a$, ce quil a pour effet de placer le procès à l'infinitif qui suit non pas en ultériorité mais en antériorité ;

- reste un point particulièrement difficile à expliquer : pourquoi, alors que venir de + $N$ ne spécifie pas la distance entre $\alpha$ et $\omega$-de sorte que je peux dire l'énoncé (11) Corinne vient de Paris, aussi bien si j'occupe un lieu proche qu'un lieu lointain de Paris, venir auxiliaire $+d e+$ Vinfinitif pose que p. ex. en (23) la distance temporelle entre venait de partir et reparut est proximale, et jamais neutre ou distale ? Différentes explications ont été avancées (i. a. Havu 2005 : 286, Honeste 2005 : 301, Bourdin 1999 : 213) qui n’emportent pas notre adhésion. Notre hypothèse, peu assurée nous le reconnaissons, est la suivante : le français dispose des auxiliaires avoir et être (+ participe passé) pour signifier l'antériorité sans plus de précision : p. ex. dans (23), si on remplace « le bateau venait de partir », par « le bateau était parti ", le procès partir est signifié comme antérieur sans que soit précisée la distance par rapport à reparaître. Signifier l'antériorité par un autre auxiliaire (venir de) tend à n'avoir de pertinence que pour la spécifier comme minime. Cette hypothèse se voit confortée par deux faits : dans les autres langues romanes, la spécification de l'antériorité d'un procès est de l'ordre du proximal, et non du distal ; en français, l'ultériorité est aussi initialement d'ordre proximal ${ }^{12}$ : «elle va le quitter » supra (17) pose la distance temporelle entre $t_{0}$ et le procès quitter comme brève.

Sur la base de leur commune origine spatiale grammaticalisée en mouvement abstrait, aller a développé les emplois narratif, ultérieur, modalisateur, et progressif du fait de sa nondéicticité, comme venir l'a fait pour l'emploi d'antériorité proximale du fait de sa déicticité.

\subsubsection{Emplois communs}

\section{L'extraordinaire}

Damourette et Pichon nomment extraordinaire l'effet de sens dans lequel l'auxiliarisation des verbes aller et venir " confère au verbe dont l'infinitif le suit un caractère dérangeant par rapport à l'ordre attendu des choses » (op. cit. : § 1652). Soit :

(25) je trouve un peu fort que des latinistes, pour garder leurs postes, viennent faire de la grammaire française. Que diraient-ils si on faisait de l'initiation au latin ou au grec ? (courrier électronique, 2009)

(26) eh ! qui se fût allé imaginer qu'un jeune paysan, sans usage du monde, (...) captivait la plus belle femme de la ville ? (Restif de La Bretonne, La Paysanne Pervertie Ou Les Dangers de La Ville, 1784).

\footnotetext{
${ }^{12}$ On sait que l’ultérieur, à la différence de l’antérieur, a pu en partie se libérer de cette contrainte de proximité.
} 
On parlera d'effet de sens modal : l'énonciateur porte un fort jugement sur le procès auxilié qu'il rejette comme inacceptable ou comme improbable : en (25), il condamne le fait que des latinistes fassent de la grammaire française à la place des linguistes ; en (26), il juge impensable qu'un jeune paysan captive la plus belle femme de la ville. Les procès à l'infinitif sont évalués comme au-delà de la limite de ce qui doit être, déontico-axiologiquement (25) ou épistémiquement (26) (Bres et Labeau 2012b) ${ }^{13}$.

Quel rôle jouent nos auxiliaires ? En posant un déplacement vers la borne initiale du procès, ils dégagent en quelque sorte un espace avant le procès lui-même, dans lequel peut venir se loger un jugement du locuteur. En appui sur le mouvement qui porte aller comme venir, le locuteur place le procès qui est atteint au terme de ce déplacement comme en dehors de ce qu'il considère comme déontico-axiologiquement ou épistémiquement attendu, en franchissement de la frontière qui sépare l'ordinaire de l'extraordinaire.

Nous proposons donc d'analyser l'extraordinaire comme résultant de l'interaction entre le mouvement abstrait vers la borne initiale du procès à l'infinitif signifié par aller et venir, et un jugement modal du locuteur.

Si aller comme venir peuvent être des ingrédients de l'extraordinaire, ils colorent différemment cet effet de sens et y participent dans des proportions inégales, du fait de leur rapport à la déicticité :

- venir impliquant une subjectivité «laisse entendre qu'on est pour ainsi dire personnellement atteint par l'acte dérangeant » (Damourette et Pichon § 1667, p. 123) ;

- aller, libre de cette assignation de subjectivité, s'avère bien plus fréquent. Notre corpus de 500 occurrences est réalisé à 82\% par aller, qui de plus n’hésite pas empiéter sur les terres déictiques de venir, comme en (27) où, plutôt que «j'irais vous dire », on attendrait « je viendrais vous dire » dans la mesure où la destination du mouvement abstrait correspond à la $2^{\text {ème }}$ personne vous :

(27) - Et après cela, j'irais vous dire qu'il m'aime ! Vous ne me le conseilleriez pas. (Marivaux P., L'Heureux stratagème, 1733)

\section{L'extrême}

D. Leeman (2005) est la première à notre connaissance à avoir relevé et décrit le tour aller jusqu'à + Vinfinitif, que l'on a dans (28-29) :

(28) Comment jugeriez- vous un homme qui irait jusqu'à trahir ses convictions pour plaire à la femme qu'il aime ? (Éluard P., Poèmes retrouvés, Préfaces, 1908-1966)

(29) Je crois qu'il est allé jusqu'à croire qu'il pouvait faire que mon mari revienne, cela pour me garder moi. (Duras M., La Douleur, 1985)

La relation avec le sens spatial est ici très lisible, du fait de la préposition jusqu'à : aller jusqu'à présuppose une série d'étapes, ici une série d'actes, au terme de laquelle est placé l'acte qui conclut la série, et qui est modalement investi comme franchissant la limite de ce qui doit être, déontico-axiologiquement (28) ou épistémiquement (29), ce qui rapproche l'extrême de l'extraordinaire.

\footnotetext{
${ }^{13}$ Ce tour s’est développé également en catalan, espagnol, italien, occitan.
} 
Comme d'ailleurs pour cet effet de sens-ci, l'extrême peut user de venir mais très rarement (on trouve plus souvent en venir jusqu'à) lorsque la destination $\omega$ se voit investie subjectivement :

(30) il n'est permis de punir le crime que quand il ne reste aucun moyen d'en douter. Mais quand on vient jusqu'à refuser d'entendre l'accusé vivant et présent, (...) quand on lui cache avec le plus grand soin l'accusation, l'accusateur, les preuves, dès lors toutes ces preuves devenues suspectes perdent toute leur force sur mon esprit. (Rousseau J.-J., Rousseau juge de Jean-Jacques, 1776)

En interaction avec certains contextes, aller et venir participent tous deux à la production des effets de sens d'extraordinaire et d'extrême sur la base du mouvement abstrait qui les définit ; l'emploi de venir est quelque peu contraint par sa déicticité, qui ajoute au jugement modal un investissement subjectif, alors que celui d'aller, à l'inverse, est favorisé par sa non-déicticité.

\section{Conclusion}

Après avoir décrit le fonctionnement des verbes aller et venir, nous avons développé l'hypothèse selon laquelle leur grammaticalisation en auxiliaires s'effectuait sur la base de leur sémantique commune de déplacement vers la destination $\omega$, et sur leur fonctionnement différent par rapport à la déicticité. Nous avons testé cette hypothèse en analysant les principaux emplois des périphrases de structure [aller / venir (+ prép.) + Vinfinitif , gérondif]. L'étude apporte de l'eau au moulin de l'hypothèse localiste: les différents sens aspectuotemporels et modaux produits par aller et venir auxiliaires trouvent leur origine dans le sémantisme spatial qui est le leur en tant que verbes de déplacement. Plus précisément : le paramètre de la déicticité permet d'expliquer pourquoi un emploi spécifique de venir comme l'antérieur immédiat est interdit à aller ; et pourquoi un emploi spécifique de aller comme le narratif, est interdit à venir. La déicticité permet également de rendre compte de la moindre productivité de l'auxiliaire venir (3 emplois spécifiques, contre 5 pour aller), comme du fait que dans les emplois qu'ils ont en commun (extraordinaire, extrême), venir est beaucoup moins fréquent que aller.

Chemin faisant, nous avons évoqué le fonctionnement des auxiliaires itif et ventif dans certaines langues romanes : il apparaît que la langue française a fait des choix communs à certaines d'entre elles, comme l'usage de aller pour l'ultérieur qu'elle partage avec l'espagnol, le portugais, l'occitan, et qui tend à se développer en italien moderne ; ou plus spécifiques comme celui de venir (de) pour l'antérieur immédiat qu'elle ne partage qu'avec l'occitan (mais qui émerge en castillan latino-américain, et dans certains dialectes de la péninsule ibérique). Le présent travail ouvre donc sur une étude systématique contrastive de l'auxiliarisation des formes itive et ventive dans les différentes langues romanes.

\section{Références bibliographiques}

Aurnague M., 2008, « Qu'est-ce qu’un verbe de déplacement ? : critères spatiaux pour une classification des verbes de déplacement intransitifs du français », $1^{\mathrm{er}}$ congrès mondial de linguistique française, www.linguistiquefrancaise.org/ , 1905-1917.

Berchem Th., 1968, "Considérations sur le parfait périphrastique vado + infinitif en catalan et gallo-roman », in Quilis A. (éd.), Actas del XIe congreso internacional de lingüística y filología románicas, vol. 3, Madrid, 1159-1170.

Bouchard, D. (1993) «Primitifs, métaphore et grammaire: les divers emplois de venir et aller », Langue française 100 : 49-66. 
Bourdin P., 1992, "Constance et inconstances de la déicticité : la resémantisation des marqueurs andatifs et ventifs ", in Morel M.-A. \& Danon-Boileau L., (éds.), La deixis. Paris : PUF, 287-307.

Bourdin P., 1999, "Venir de et la récence : un marqueur typologiquement surdéterminé », Cahiers Chronos 4, 203-231.

Bourdin P., 2005, «Venir en français contemporain: de deux fonctionnements périphrastiques », in Bat-Zeev Shyldkrot H. et Le Querler N. (éds), Les périphrases verbales. Philadelphia: Benjamins, 261-278.

Bourdin P., 2008, "On the grammaticalization of 'come' and 'go' into markers of textual connectivity”, in Lopez-Couso M.J. \& Seoane E. (eds), Rethinking grammaticalization: New Perspectives. Philadelphia: John Benjamins, 37-59.

Bres J. \& Barceló G.J., 2007, « La grammaticalisation de la forme itive comme prospectif dans les langues romanes », in Fernandez-Vest, J. (ed.). Combat pour les langues du monde/Fighting for the world's languages : Hommage à Claude Hagège. Paris :

L'Harmattan, 91-104.

Bres J. \& Labeau E., 2012a, « De la grammaticalisation des formes itive (aller) et ventive (venir) : valeur en langue et emplois en discours », in Saussure L. de \& Rihs A. (éd.). Etudes de sémantique et pragmatique françaises, Berne : Peter Lang, 143-165.

Bres J. \& Labeau E., 2012b, «Allez donc sortir des sentiers battus! La production de l'effet de sens extraordinaire par aller et venir », Journal of French Language Studies, 1-27. http://journals.cambridge.org/action/displayJournal?jid=JFL

Bres J. \& Labeau E., 2012c, « Un phénix linguistique ? Le tour narratif va + infinitif renaîtrait-il, en français contemporain, de ses cendres médiévales ? », in C. Guillot C., Combettes B., Lavrentiev A., Oppermann-Marsaux E. \& Prévost S., (éds.), Le changement en français. Bruxelles : Peter Lang, 1-14.

Bres J. \& Labeau E., 2013a, “About the Illustrative use of the aller infinitive periphrasis in French”, in Labeau, E. \& Bres, J. (eds), Evolution of romance tenses, Bruxelles : Peter Lang, sous presse, 157-185.

Bres J. \& Labeau E., 2013b, "Venir de + infinitif: grammaticalization of come into an aspectuo-temporal auxiliary and its defectivity in French" (soumis).

Bybee J., Perkins R., \& Pagliuca W., 1994, The evolution of grammar, Chicago: The University of Chicago Press.

Chevalier J.-C., 1976, « Sur l'idée d'aller et de venir, et sa traduction linguistique en espagnol et en français », Bulletin hispanique, LXXVIII, n³-4, 254-312 .

Colón G., 1976, «Sobre el perfet perifràstic vado + infinitiu en català, en provençal i en frances ", in Problemes de llengua i literatura catalanes, Actes del II colloqui internacional sobre el català (Amsterdam 1970). Barcelona: Publicacions de l'Abadia de Montserrat,101144.

Dahl Ö., 2000, "The tense-aspect systems of European languages in a typological perspective" in Dahl, Ö. (ed.), Tense and Aspect in the Languages of Europe. Berlin / New York: Mouton de Gruyter, 3-25.

Damourette, J. \& Pichon, J., 1911-1936/1970, Des mots à la pensée : Essai de grammaire de la langue française (Tome 5). Paris : D’Artrey.

De Mulder W., 2010, "La métaphore espace/temps à l'épreuve : l'évolution de venir de », Cahiers Chronos 21, 65-83.

Dominicy M., 1983, “Time, tense and restriction (on the French periphrasis venir de + infinitive)”, Communication \& Cognition 16, 1/2:133-154.

Fillmore Ch., 1997 (1967/1975), Lectures on Deixis, Stanford: CSLI Publications. (Version initiale : Santa Cruz Lectures on Deixis, 1975/1971). 
Fleischman S., 1982a, “The past and the future: are they coming or going?”, Berkeley Linguistics Society 8: 322-334.

Fleichman S., 1982b, The Future in Thought and Language: Diachronic Evidence from Romance. Cambridge: Cambridge University Press.

García Fernández L., 2006, (dir.), Diccionario de perífrasis verbales. Madrid : Gredos.

Gougenheim G. 1929 / 1971. Etudes sur les périphrases verbales de la langue française. Paris : Nizet.

Guillaume G., 1929/1970. Temps et verbe. Paris : Champion.

Hagège Cl., 1993, The language builder. Amsterdam: John Benjamins.

Havu J., 2005, «L'expression du passé récent en français: observations sur l'emploi de la périphrase venir + INFINITIF », in Bat-Zeev Shyldkrot H. \& Le Querler N. (éds), Les périphrases verbales. Philadelphia: Benjamins, 279-292.

Heine B. 2003, “Grammaticalization”, in Joseph B. \& Janda R.,The Handbook of Historical Linguistics. Oxford: Blackwell, 575-601.

Honeste M.-L., 2005, "Venir est-il un verbe périphrastique? », in Bat-Zeev Shyldkrot H. \& Le Querler N. (éds.), Les périphrases verbales. Amsterdam / Philadelphia: John Benjamins, 293-310.

Hopper P. \& Traugott E., 1993/2003, Grammaticalization. Cambridge: Cambridge University Press.

Kerbrat-Orecchioni C., 1980, L'énonciation de la subjectivité dans le langage, Paris : A. Colin.

Labeau E., 2012a, « Une façon d'indiquer la 'non-coïncidence entre les mots et les choses', on va dire... ", $3^{\text {ème }}$ Congrès Mondial de Linguistique Française, www.linguistiquefrancaise.org/, 573 - 582.

Labeau, E., 2012b, “A linguistic resurrection? Aller + infinitive in narrative past contexts”, in Lagorgette, D \& Pooley, T. (eds), On linguistic change in French: socio-historical approaches / Le changement linguistique en français: aspects socio-historiques, Studies in honour of R. Anthony Lodge / Études en hommage au Professeur R. Anthony Lodge, Chambéry : Presses de l’Université de Savoie, 85-98.

Lamiroy B., 1983, Les verbes de mouvement en français et en espagnol. Amsterdam: John Benjamins Publishing Company.

Langacker R., 1987, « Mouvement abstrait », Langue française 76, 59-76.

Lansari L., 2009, Les périphrases verbales aller + inf. et be going to. Paris : Ophrys.

Lansari, L., 2010, "On va dire : vers un emploi modalisant d'aller + infinitif », Cahiers Chronos 21, 119-139.

Larreya. P., 2005, "Sur les emplois de la périphrase aller + infinitif », in Bat-Zeev Shyldkrot H. \& Le Querler N. (éds.), Les périphrases verbales. Amsterdam / Philadelphia: John Benjamins, 337-360.

Leeman D., 2005, «Un nouvel auxiliaire : aller jusqu'à », in Bat-Zeev Shyldkrot H. \& Le Querler N. (éds.), Les périphrases verbales. Amsterdam / Philadelphia: John Benjamins, 361377.

Lyons J., 1977, Semantics. Cambridge: Cambridge University Press.

Meillet A., 1912/1926, « L'évolution des formes grammaticales », in Linguistique historique et linguistique générale, Paris : Champion, 130-148.

Narrog H. et Heine B., 2011 (dir.), Oxford Handbook of Grammaticalization. Oxford: Oxford University Press.

Schøsler L., 2005, «Grammaticalisation et dégrammaticalisation. Etude des constructions progressives en français du type Pierre va / vient / est chantant », Cahiers Chronos 16, 91119. 
Squartini M., 1998, Verbal periphrases in Romance: Aspect, Actionality and Grammaticalization. Berlin: Mouton de Gruyter.

Steuckardt A., 2012, " Polyphonie et médiativité dans un marqueur émergent : on va dire », communication au colloque international Les marqueurs du discours : polyphonie, modalité et médiativité en français, Universidad Complutense de Madrid, Madrid 22-23 octobre.

Vetters C., 2010, «Développement et évolution des temps du passé en français: passé simple, passé composé et venir de + infinitif », Cahiers Chronos 21, 277-298. 
